\title{
Prospects for organic minerals on saturn's moon titan
}

Helen Elizabeth Maynard-Casely ${ }^{1}$, Morgan Cable ${ }^{2}$, Mike Malaska ${ }^{2}$, Tuan Vu², Mathieu Choukroun ${ }^{2}$, Rob Hodyss ${ }^{2}$

${ }^{1}$ Australian Nuclear Science And Technology Organisation, Kirrawee, Australia, ${ }^{2}$ Jet Propulsion Laboratory, Pasadena, United States E-mail: helenmc@ansto.gov.au

Titan, the largest moon of Saturn, contains a vast inventory of organic molecules and is considered a prebiotic chemical laboratory on a planetary scale. Active photochemistry in the atmosphere via solar radiation and energy from Saturn's magnetosphere causes nitrogen and methane to dissociate and recombine, generating organics ranging from simple (ethane, acetylene, $\mathrm{HCN}$ ) to complex ( $>10,000 \mathrm{Da})$ molecules. These molecules continue to react as they move through Titan's atmosphere, forming aerosol haze layers and eventually depositing on the surface [1].

Additionally, the Cassini spacecraft revealed that Titan has standing bodies of liquid on its surface, in the form of lakes and seas. This is a remarkable discovery, as it makes Titan only the second planetary body known to have such features (after our own Earth). These lakes, which are evidenced to contain mainly methane and ethane, could dissolve many of the molecules that were generated in Titan's atmosphere. These could subsequently form precipitates and create evaporite deposits similar to those observed by the Cassini Visual and Infrared Mapping Spectrometer (VIMS) and Synthetic Aperture Radar (SAR) around some of the northern lakes [2]. Previous work has demonstrated [3] that two common organic molecules on Titan, ethane and benzene, form a unique and stable co-crystalline structure at Titan surface temperatures, which could comprise these evaporite deposits.

Influenced by the discovery of a new solid phase for Titan, a survey has been undertaken outlining the current structural understanding of molecular solids under Titan conditions. Using the Cambridge Structural Database (CSD) a number of possible minerals 'types' that would be expected on the surface of Titan have been identified. The subsequent classification of possible Titan minerals is done on the basis of intermolecular interactions, with the materials organized into 'Molecular solids', 'Molecular co-crystals' and 'Hydrates' grouping. This classification is designed to aid future work in determining how a number of the features on Titan may have formed.

[1] Cable, M.L. et al. (2012) Chem. Rev., 112, 1882-1909

[2] Barnes, J.W. et al. (2009) Icarus, 201, 217-225.

[3] Vu, T.H. et al. (2014) J. Phys. Chem. A, 118, 4087-4094, Cable, M.L. et al. (2014) GRL, 41, 5396-5401, Maynard-Casely, H.E. et al. (2016) IUCrJ, 3, 192-199

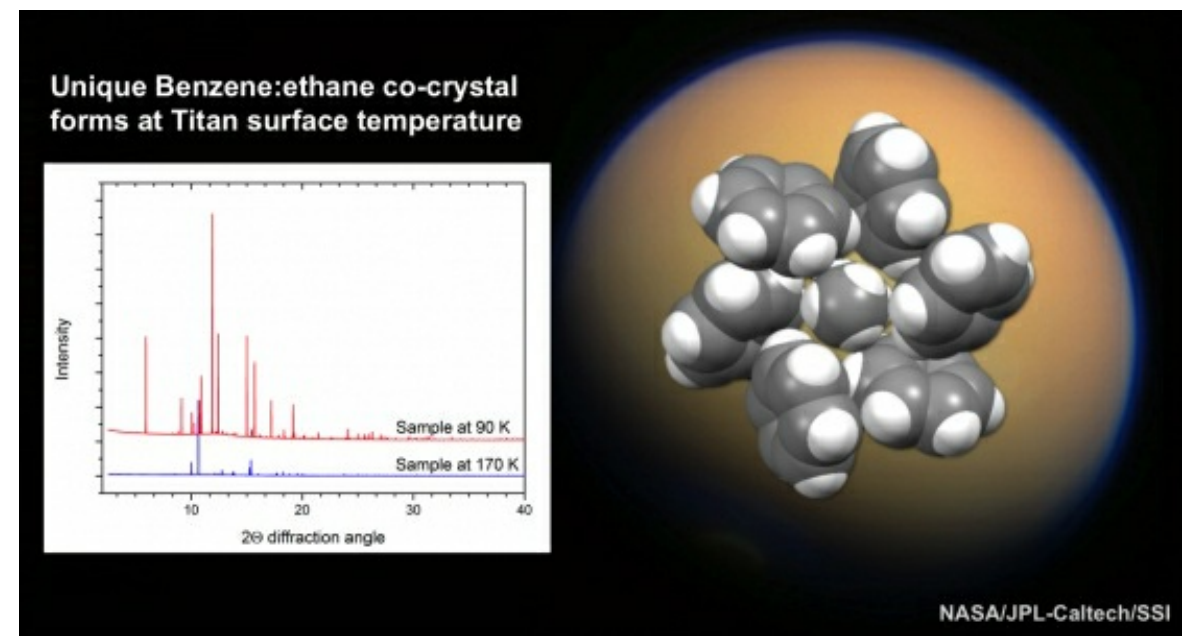

Keywords: Minerals, Organic, planetary 